\title{
EL PAPEL DE LA PARADIPLOMACIA ENTRE EL NORTE DE CHILE Y EL SUR DEL PERÚ: ANTECEDENTES HISTÓRICOS, LIMITACIONES INSTITUCIONALES Y NUEVOS DESAFÍOS "POSFALLO DE LA HAYA"
}

\author{
THE ROLE OF PARADIPLOMACY BETWEEN THE NORTH OF CHILE AND \\ THE SOUTH OF PERU: HISTORY, INSTITUTIONAL RESTRICTIONS AND NEW \\ CHALLENGES "POST LA HAYA VERDICT”
}

Cristian Ovando Santana* y Sergio González Miranda**

\begin{abstract}
Tacna y Arica tienen una historia común colonial y republicana innegables, sin embargo, después del Tratado de Ancón de 1884 y hasta la actualidad, donde el fallo de La Haya sobre el límite marítimo ha sido el último litigio, los Estados nacionales de Perú y Chile han tensionado las relaciones fronterizas hasta los límites de la diplomacia. Aquí se analiza el papel paradiplomático que han desempeñado actores de las sociedades de Tacna y Arica, con el propósito de alcanzar una paz social que les beneficie, aprovechando la complementariedad económica del valle (Tacna) y del puerto (Arica).

Mediante la puesta en marcha de diversos acuerdos vecinales transfronterizos, para atender aspectos de cooperación local específicos, los actores paradiplomáticos tacnoariqueños han construido una identidad transfronteriza, desafiando incluso algunas prácticas nacionalistas.
\end{abstract}

Palabras claves: Tacna y Arica, historia común, pluralismo diplomático y paradiplomacia, posfallo de La Haya, sociedad civil.

Tacna and Arica have a mutual irrefutable colonial and republican history; however, after the Treaty of Ancon (1884) and until now, in which La Haya verdict on water borders has been the last law suit, the nation states of Peru and Chile have put pressure on border relationships until the limits of diplomacy. Here the paradiplomatic role is analyzed that actors of the societies of Tacna and Arica have performed, with the purpose of achieving a social harmony to benefit them, taking advantage of the economic complementarity of the valley (Tacna) and the port (Arica).

Through the implementation of different cross-border neighboring agreements, to deal with specific local cooperative aspects, paradiplomatic actors from Tacna-Arica have built a cross-border identity, challenging even some nationalist practices.

Key words: Tacna Arica, mutual history, diplomatic pluralism and paradiplomacy, post La Haya ruling, civil society.

\section{Introducción}

Este artículo profundiza en las posibilidades de algunas actividades paradiplomáticas, particularmente las emprendidas desde las agencias de frontera y los respectivos consulados, teniendo en cuenta algunos antecedentes históricos en torno a los alcances regionales de los litigios diplomáticos acontecidos entre Perú y Chile, destacando además ciertas limitaciones institucionales y los nuevos desafíos que emprenden tras el fallo de $\mathrm{La}$ Haya. Respecto de estos últimos, abordaremos las iniciativas que han empezado a dibujar algunos actores paradiplomáticos de Tacna y Arica en colaboración con los consulados de ambos países $\mathrm{y}$ algunas universidades, quienes intentan sortear las consecuencias negativas del litigio de La Haya mediante la puesta en marcha de diversos acuerdos vecinales transfronterizos. Con ellos buscaremos abordar propuestas de cooperación bilateral en temas de variada índole. Planteamos que estos actores han construido una identidad trasfronteriza por medio de iniciativas emprendidas entre las ciudades de Tacna y Arica, las que además estarían silenciosamente incidiendo en el quehacer diplomático que emprenden los cuerpos consulares, al punto que, sin ser su objetivo deliberado, contribuyen a la descentralización del sistema diplomático y de esta forma estarían tendiendo a la búsqueda de un mayor compromiso con el otro diplomático, el extranjero.

\footnotetext{
* Universidad Arturo Prat, Instituto de Estudios Internacionales. Iquique, Chile. Correo electrónico: covando@unap.cl

** Universidad de Tarapacá, Departamento de Ciencias Históricas y Geográficas. Arica, Chile. Correo electrónico: pampino50@ gmail.com
} 
Previamente desarrollaremos algunos antecedentes históricos de los vínculos chileno-peruanos que dan cuenta de cómo los Estados nacionales de Perú y Chile han tensionado las relaciones fronterizas hasta los límites de la diplomacia, aunque resistidas por sus regiones fronterizas, quienes tienen su propia lógica cotidiana acerca de estos incidentes . A continuación desarrollaremos algunos aspectos teóricos de la paradiplomacia, en el marco de la apertura de los estudios diplomáticos, distinguiéndose este enfoque del que aporta la actuación de los gobiernos no centrales.

\section{Contexto histórico: incidentes diplomáticos y su proyección regional}

Históricamente, mientras las diplomacias de Perú y Chile han estado en proceso de resolver sus litigios fronterizos (González 2006, 2008), las ciudades de ambos países que comparten frontera, Tacna y Arica, han seguido su dinámica de constante trasiego, cooperación e intercambios en múltiples dimensiones (González 2006; Graña 2001; Tapia, 2014, 2015; Dilla 2016).

Esta dinámica transfronteriza se expresa de tal manera que ocasionales diferencias entre Lima y Santiago parecieran no mermarla (Ovando y González 2014; Dilla 2016). Pese a ello, esta misma pareció verse afectada por la demanda peruana interpuesta en 2012 en contra de Chile, ante el Corte Internacional de Justicia de La Haya, en adelante CIJ, a causa de la supuesta inexistencia de un acuerdo bilateral que regulase la frontera marítima. Posteriormente, varias iniciativas del gobierno peruano han acentuado la tensión fronteriza, como la denominación oficial de "El Mar de Grau"1, y la creación del distrito peruano de Yarada-Los Palos, ubicado en el denominado "triángulo terrestre", el supuestamente último residual post-La Haya.

Estos acontecimientos han motivado que se involucren ciertos actores paradiplomáticos de Tacna y Arica quienes, por medio de algunas iniciativas transfronterizas, buscaron y siguen buscando un mayor entendimiento entre ambas ciudades. Mediante ellas intentan morigerar los efectos negativos del fallo de la CIJ, especialmente la incitación a la opinión pública regional, que se vio bombardeada diariamente por una prensa que exacerbaba el litigio, junto con destacar cómo estos mismos actores han contribuido a fortalecer el desarrollo regional transfronterizo con diversas iniciativas pioneras, que repasaremos a lo largo de este trabajo.

En suma, ante un mismo hecho se desplegaron dos sentidos de la frontera, la geopolítica, desde las capitales, y la de la vida cotidiana, desde el epicentro del litigio, con sus expresiones contradictorias (Dilla 2016:311,312 y ss.).

Una de las principales consecuencias derivadas de la posguerra del Pacífico es el surgimiento de un juego diplomático caracterizado por la estrategia chilena de retener Tacna y Arica como un territorio contenedor con Perú (que alejara la frontera de su principal industria de la época: el nitrato de soda), o, de posible negociación futura con Bolivia. La estrategia peruana, por su parte, era considerar irrenunciable estos territorios generando un sentimiento irrendentista entre su población originaria (González 2004, 2008). Este periodo crítico se prolongó por cuarenta y cinco años ${ }^{2}$, lo que permitió a escala regional, pero sobre todo a escala local, "normalizar" los efectos de la crisis diplomática, posibilitando una vida cotidiana entre poblaciones "supuestamente" en pugna. Un ejemplo de lo anterior se refleja en el estudio realizado por Skuban (2007). Este autor recurre al concepto de hegemonía de corte gramsciano, donde la acción hegemónica chilena habría logrado atraer adhesión de población peruana, especialmente indígena. Aquí surge lo que Skuban denomina la "cuestión indígena” (2007:102), que presentó un problema para las autoridades peruanas, las que dudaron del nacionalismo de estas porque las comunidades andinas no tendrían "otro Dios y otra Patria que su chacra" (Skuban 2007:101). Sin embargo, este autor no identifica una "cuestión empresarial" cuando los productores agropecuarios se adaptaron al mercado chileno, como señala Alfredo Kapsoli: "En un primer momento se mostraron nacionalistas, juraron perder bienes y vidas en defensa de la Patria; pero apenas los chilenos pisaron tierra peruana abjuraron de esos principios y terminaron colaborando con nuestros enemigos. Sus intereses de clase primaron sobre el problema nacional" (Citado por Panty Neira et al. 2001:163).

Estos autores desconocen que hubo un espacio tacnoariqueño integrado desde el siglo XVIII a redes comerciales y empresariales, especialmente agropecuarias y mineras (Rosenblitt 2010), las que llegaron hasta Tarapacá por la importancia de sus minas de plata, primero, y salitre, después. Desde el inicio de la administración chilena de Tarapacá, 
Tacna y Arica nunca dejaron de estar vinculadas al mercado salitrero, entre otras razones, porque algunos flujos bolivianos de bienes, mercancías y trabajadores utilizaron la antigua ruta entre Arica y Potosí. Tacna proporcionaba también mercancías elaboradas (González 2006) y algunas de las más importantes compañías salitreras tenían su casa matriz en esa ciudad, como fue el caso de Campbell, Outram y $\mathrm{C}^{\circ}$, dueña de oficinas de gran tamaño como Agua Santa y San Antonio de Zapiga.

La perspectiva nacionalista de los propios autores impide observar que esos actores y sus acciones pueden ser calificadas de paradiplomáticas, siguiendo la interpretación genealógica que Der Derian (1987) hace de este neologismo - para: "junto a", "al lado de", pero también "divergente de", "opuesto a"-, la paradiplomacia podría entonces referirse a toda forma de actividad internacional llevada a cabo por actores no tradicionales, incluyendo entre estos las corporaciones transnacionales, las organizaciones internacionales de trabajadores, las comunidades religiosas, los organismos no gubernamentales, la industria, los medios de comunicación (Carreón 2007) y, por cierto, empresarios, campesinos, trabajadores e indígenas, que circulan por las fronteras internacionales, como veremos en apartado teórico más adelante. El peso de la historia de esas redes comerciales transfronterizas no puede quedar al margen de nuevas reflexiones en torno a la paradiplomacia en el espacio tacnoariqueño. La perspectiva nacionalista, en cambio, reduce un fenómeno complejo a la dualidad amigo/enemigo (Schmitt 2005) y se concentra en la escala del Estado-Nación.

El escritor Freddy Gambetta cuenta en su Nueva crónica del tiempo viejo, que fue criado por "dos señoritas de Tacna"3, las que, al recordar ese periodo de la ocupación chilena, "eran discretas en sus comentarios, contaban las historias buenas y malas, sin exageraciones, sin fabricar mitos. Reconocían, por ejemplo, la excelente educación que habían recibido de los maestros chilenos, el sentido de la responsabilidad, del aseo, de la puntualidad y la honradez" (2001:14). Curiosamente, el reconocido historiador Jorge Basadre, al escribir acerca de su "infancia en Tacna", recuerda con cariño a su maestra peruana que utilizaba el libro chileno Silabario Matte (Basadre 1975).

La vida cotidiana ${ }^{4}$ termina por imponerse a la sensación de crisis, aunque se pueda constatar la existencia de violencia política, sea expresada en el imaginario (Galdames et al. 2014), en el discurso (2014), en lo religioso (Choque 2014) o sociopolítico (Mondaca et al. 2014), incluso en la acción (Soto et al. 2014, Díaz et al. 2014). Por tanto, lo que la memoria registra son incidentes y, como están relacionados a un litigio diplomático, podríamos calificarlos de incidentes con repercusiones sociales (Cornago 2014), porque si bien los incidentes diplomáticos nos recuerdan que ellos descansan en la ficción última de la soberanía de los Estados, nos evocan, además, que las relaciones diplomáticas son instancias sociales, por lo que se deben a la sociedad en su conjunto, quien ha delegado en las autoridades su representatividad. De tal manera que la importancia del incidente diplomático radica en que sus efectos impregnan el cuerpo social, además que las sociedades -en nuestro caso regionales y epicentro de una guerra- siempre han encontrado en el estallido ocasional de incidentes diplomáticos la oportunidad de hacer oír su voz (Cornago 2014).

En suma, así como aconteció en años de mayor violencia política producto de la tensión diplomática en la pos-Guerra del Pacífico, en el periodo denominado "plebiscitario" (1902, 1908, 1910, 1911, 1919, 1927), observamos que también a consecuencia del fallo de la CIJ, algunas agrupaciones nacionalistas tacneñas llamaron a realizar manifestaciones en la frontera ${ }^{5}$. Sin embargo, la vida cotidiana fronteriza ha prevalecido permitiendo la emergencia de redes transfronterizas, algunas asociadas a expresiones culturales como las cofradías, educativas como las escuelas de frontera, comerciales como las ferias al menudeo, familiares como los apellidos y linajes similares en Tacna y Arica, municipales como las acciones integracionistas de los alcaldes de ambas ciudades y la Alianza Estratégica Aymaras Sin Fronteras $^{6}$, entre otras.

\section{Paradiplomacia en el marco del debate acerca de pluralismo diplomático}

En el debate de la teoría de las relaciones internacionales que estudia a los actores subnacionales, está pendiente una teorización de la paradiplomacia entendida desde su "relevancia ampliada". Considerar la paradiplomacia acotada a la actuación de los agentes gubernamentales subestatales, paralelos a las relaciones exteriores de los Estados, y excluir los actores no estatales ubicados en la escala regional-local parece muy simplificador. Amilhat Szary $(2016: 47,48)$ al 
respecto señala categóricamente: “ ... el alcance de la paradiplomacia concierne a un número cada día más creciente de personas, junto con incorporar una cantidad igualmente relevante de actores de gran diversidad; por ello su relevancia ampliada impone un examen teórico (...), de forma de llegar a proponer una redefinición crítica del fenómeno paradiplomático (Amhilat Szary,).

Desde esta premisa, en las últimas dos décadas surge dentro del debate respecto de la ampliación de la paradiplomacia una corriente crítica de la diplomacia que va más allá de la discusión de aquella y que se posiciona respecto del estatus ontológico de la misma. Es posible destacar que a esta corriente le anteceden, dentro de la teoría de las relaciones internacionales, posiciones neofederalistas y transnacionalistas, de las que se desprende el origen del término paradiplomacia acotado a los actores subestatales o gobiernos no centrales dentro de Estados federales (Duchacek, 1984, 1986; Keating y Aldecoa, 1999). Este debate, que precedió al que abordamos en este trabajo, se centró en el impacto del sistema internacional en la gestión de la política exterior y su ajuste debido a la intromisión de los gobiernos no centrales en los asuntos internacionales de su competencia (Ugalde, 2005: 296 y ss.).

En cambio, la postura crítica que guía esta investigación cuestiona la idea de que la diplomacia esté reserva solo al Estado, al Ministerio de Relaciones Exteriores y a sus agentes autorizados, aunque estuvieren ubicados a distinta escala (Constantinou 2013). Este enfoque plantea que "es posible que el Estado defina los objetivos que quiera conseguir en política internacional y, al mismo tiempo, que la negociación pueda ser realizada por actores distintos de los diplomáticos del Ministerio de Asuntos Exteriores" (Cobino 2005: 2). Siguiendo este argumento, plantea superar la idea de que se trata de un arte "extremadamente formalizado y rígido, dentro de una más amplia maquinaria de la política exterior del Estado, completamente aislado de las experiencias sociales reales, y privado de cualquier relevancia política inmediata de nuestra vida diaria" (Cornago 2013b: 7-18). Surge así una idea renovada acerca de la diplomacia que toma en cuenta la subjetividad de cada actor que reivindica un rol internacional y el contexto histórico en que opera. Así, esta aproximación nos permite constatar evidencias que den cuenta de espacios "para el entendimiento diplomático en las más diversas expresiones de la vida social" (Cornago 2013b:11). Se trata de una renovada idea de diplomacia entendida como homodiplomacia (Durán 2012; Constantinou y Der Derian 2010). Una alternativa a la diplomacia centrada en la práctica estatal, oficial. Su crítica apunta además a que el enfoque tradicional estadocéntrico "tiende a reproducir la ficción de la existencia de una comunidad política perfecta (...) como la asunción fundamental que da sentido a todo el sistema de reconocimiento diplomático, la representación y la negociación entre los Estados (Cornago 2015: 6). En cambio, la homodiplomacia destaca las características intrínsecas de los territorios en que operan actividades paradiplomáticas y que no siempre coinciden con los contornos y límites de Estado-Nación, junto con revelar los aspectos transformativos de la diplomacia como la mediación de los iguales allende la frontera (Durán 2012). Constantinou llama homodiplomacia a una forma de diplomacia centrada en la homología entre el uno mismo y el otro, el extranjero (Durán 2012). Siguiendo esta postura, Durán (2012) busca por medio de este enfoque destacar lo que los une como parte de un territorio más amplio del que hace referencia lo estatal-nacional, a partir de la constitución mutua de los sujetos ${ }^{8}$ desde rasgos identitarios que experimentan en aquel espacio transfronterizo. Con ello se pretende proporcionar una mayor comprensión respecto de los motivos y los resultados de la diplomacia subnacional, más allá de los aspectos funcionales que destaca la paradiplomacia más tradicional, como es el caso de las motivaciones económicas para emprender estas actividades, destacando en cambio aspectos subjetivos de la relación.

Con ello se pretende proporcionar una mayor comprensión respecto de los motivos y los resultados de la diplomacia subnacional, toda vez que esta dimensión identitaria de base territorial, en torno a la alteridad, es clave para comprender intercambios societales en contextos transfronterizos y que fueron epicentro de un conflicto que perdura hasta la fecha, como ocurre en nuestro caso de estudio.

Desde este enfoque crítico, nos referimos a las prácticas diplomáticas -desde una comprensión normativa de aquella a partir de eventos singulares- que tomen en cuenta las implicaciones de su compromiso con el "otro" diplomático, con el que obligatoriamente se debe convivir, pero no se puede absorber (Cornago 2013: 33-34). 
Aludimos a una diplomacia entendida como el mecanismo en el que una pluralidad de actores en juego, a distinta escala, se expresan para lograr el entendimiento humano y la reconciliación política (Cornago 2013b). Esa dimensión intersubjetiva de la diplomacia, “...exige a cada parte envuelta en la relación, frente a un interlocutor que no puede ser ignorado ni vencido, el establecimiento y cultivo incesante de una relación mutua, inevitable y duradera (De Certeau 1997)" (Cornago 2016:21). Esta posibilidad estaría dada a partir de evidencias de prácticas de comunicación entre comunidades tal como se han experimentado a lo largo de la historia en diferentes contextos locales (Cornago 2009). Prácticas que, siguiendo las evidencias mencionadas, le antecede o se han desarrollado de forma paralela, pero invisibilizada.

Uno de los aspectos importantes de esta corriente, a partir de la informalidad propia de la práctica paradiplomática, que no está constreñida a un marco jurídico exhaustivo, se refiere a las promisorias posibilidades de entendimiento mutuo entre actores extraños, posibilidad que se ve limitada en las negociaciones ceñidas a rutinas diplomáticas convencionales, en la medida que sus conductas deben limitarse a dichos encuadramientos. Al respecto Cornago enfáticamente señala que algunos cientistas sociales:

(...) han enfatizado las virtudes de otras estrategias menos formales de la negociación. B.D. Cohen, por ejemplo, sostiene que mientras la negociación formal entre Estados implica la preparación de participantes de manera que los encuentros diplomáticos pueden ser breves y rutinarios, con el propósito de prevenir una expresión de sentimientos personales y posiciones en consideración con un asunto particular, los encuentros informales son más "abiertos para la autorrevelación... permitiendo a los participantes el poder liberarse ellos mismos de sus propias creencias estereotipadas", consecuentemente permitiendo que asuntos políticos sustanciales puedan ser abordados de una manera más constructiva y de una manera recíprocamente satisfactoria (Cornago, 2013: 8 y 9).

A partir de esta posibilidad de emprender negociones recíprocamente satisfactorias, más allá de una lógica utilitarista, estos enfoques permiten poner relevancia en variables culturales desde el punto de vista de la alteridad, para superar el extrañamiento entre las sociedades. Profundizan en cómo distintas unidades políticas, a partir de sus vínculos diplomáticos en un sentido pluralista, pueden reflejar de manera compartida una identidad, ideas y una determinada visión del mundo. De este modo se supera la visión fatalista, estatista y restringida de la diplomacia tradicional.

\subsection{Motivaciones de los actores paradiplomáticos de frontera}

En el ámbito socio cultural, dimensión de nuestro caso de estudio, la paradiplomacia promueve el intercambio cultural entre ciudades de regiones fronterizas, materializándose en "alianzas para dar a conocer la cultura local, con el fin de intercambiar ideas que validen estas nuevas escalas de acción internacional (...); junto con la construcción de acuerdos vecinales transfronterizos para atender aspectos de gestión y cooperación local específicos" (Luna y Ballesteros 2005:27-28). En consecuencia, la paradiplomacia en esta dimensión transfronteriza aporta dentro de sus distintas vertientes de interpretación, una nueva mirada a las relaciones internacionales de las regiones que, sin cuestionar el exclusivo poder del Estado central para definir la política exterior del país, abre nuevas posibilidades de entendimiento entre las sociedades. Consideramos que también ofrece nuevas alternativas de desarrollo: a) aprovechando nuevas oportunidades del comercio fronterizo b) aumentando la seguridad y la paz mediante la cooperación descentralizada (Marteles 2009), c) permitiendo la emergencia de plataformas pivotales (Boisier 2003) en áreas transfronterizas y corredores internacionales, d) permitiendo la formación de redes sociales transfronterizas, incluyendo las étnicas y familiares, que aumentan la densidad cultural (González, 2006), e) el control (por medio de una cooperación bi o trilateral de los gobiernos) de flujos negativos en la frontera como el narcotráfico, narcoterrorismo, contrabando, trata de personas, etc., que estigmatizan a las zonas de frontera por el miedo que estos actores ejercen (Rhi-Sausi y Oddone 2013), f) la creación de escuelas fronterizas con currículum pertinentes, como aquellas organizadas por Convenio Andrés Bello por intermedio del programa Aula Viva (Martínez 1997). 


\subsection{El papel de la paradiplomacia entre el norte de Chile y el sur del Perú}

Reflexionar acerca del rol de la paradiplomacia entre las sociedades chilenas y peruanas -desde la perspectiva chilena- nos lleva necesariamente a plantear algunas premisas.

Una de ellas es que Chile posee un marco institucional limitado ${ }^{9}$ para promover la acción exterior y transfronterizo de sus regiones (Gallardo 2006; Ovando 2013). Es factible destacar que tuvo un impulso importante con la creación de la $\operatorname{DICORE}^{10}$ (2003) y el apoyo de la SUBDERE ${ }^{11}$ en materia de mejorar la gestión internacional de las regiones chilenas. Aunque este apoyo inicial fue decayendo en el tiempo ${ }^{12}$, sobre todo en las regiones del norte grande de Chile, habida cuenta de los litigios diplomáticos que surgen con Bolivia y Perú ${ }^{13}, \operatorname{los}$ que desincentivaron el apoyo estatal a estas actividades. No obstante, dentro de los desafíos para profundizar la descentralización, la comisión asesora presidencial plantea promover Acciones de Participación Ciudadana que permitan ir incorporando a la ciudadanía en acciones que favorezcan la diplomacia social. Aunque se destaca, como veremos más adelante, el rol que han cumplido los Consulados de Chile y Perú en Tacna y Arica, respectivamente, para dinamizar las actividades transfronterizas entre actores sociales de ambas ciudades, teniendo en cuenta que la DICORE, entre otras cosas, permite vincular a los gobiernos regionales y locales con las direcciones del Ministerio de Relaciones Exteriores y consulados.

Expresión de este marco limitado constituye su escasa participación en materias fronterizas contempladas en conglomerados de integración como MERCOSUR y La Comunidad Andina (CAN). En efecto, la decisión $\mathrm{N}^{\mathrm{o}} 666$ que regula el rol de Chile en la CAN no contempla el desarrollo fronterizo como prioridad. Chile no participa en los Centros Binacionales de Atención en Frontera (CEBAF); tampoco en Banco de Proyectos de Integración y Desarrollo Fronterizo (BPIF), que financia gestión ambiental transfronteriza, por ejemplo; y no lo hace en las zonas de integración fronteriza (ZIF). Desde un punto de vista fenomenológico, la participación o no en regímenes de integración es clave en la conformación de una identidad fronteriza en materia de política exterior. En el caso del Perú, que sí participa en los órganos aludidos, dentro de su Ministerio de Relaciones Exteriores, la autoridad en materia de fronteras se denomina Dirección de Desarrollo e Integración Fronteriza; en Chile, en cambio, Dirección de Fronteras y Límites, DIFROL, enunciado que implica una labor más acotada hacia las fronteras. Por ello, equiparar los resultados de la labor de la DIFROL (por los comités de frontera, último en 2011, XII) y el resultado de las dinámicas propias de la ZIF en materia de desarrollo fronterizo, es discutible.

Además, destacamos, como ya señalamos someramente más arriba, las ocasionales tensiones diplomáticas en que se ven enfrentados las cancillerías chilenas, bolivianas y peruanas, las que también desincentivan las políticas hacia el desarrollo fronterizo. Por ejemplo, en 2012 el gobierno boliviano suspendió indefinidamente su participación en la XIII reunión del Comité de Frontera e Integración Chile-Bolivia, en la XIII reunión del Grupo de Trabajo sobre Libre Tránsito, y en la XXII Reunión de la Comisión Administradora del Acuerdo de Complementación Económica N 22 (ACE 22).

La tercera premisa dice relación con que necesariamente pensar la integración chileno-peruana desde la perspectiva del pluralismo diplomático, en el que se inscribe la noción de paradiplomacia que usamos, conlleva pensar los vínculos con Bolivia.

Señaladas estas premisas, a continuación nos referiremos al rol de la paradiplomacia en la relación bilateral chileno peruana en el contexto actual marcado por el posfallo de La Haya:

Una de los elementos que se destaca del esfuerzo actual que hacen los actores paradiplomáticos del norte de Chile, el sur del Perú y el centro oeste de Bolivia, es que no siguen la lógica de la diplomacia de las respectivas capitales, guiados en ocasiones por la opacidad, el secreto, el juego de suma cero y la cooperación débil. Constantinou (2013) habla de una diplomacia guiada por estratagemas e inspirada en la defensa irrestricta de los autodefinidos intereses nacionales ${ }^{14}$ (Constantinou y Der Derian 2010). En concreto, estaría condicionada en la actualidad por las diferencias en torno al fallo emitido por la Corte de La Haya y por polémicas derivadas del mismo. Junto con ello, dichas dinámicas están fuertemente condicionadas por la particularidad que tienen las relaciones transfronterizas que se han configurado históricamente entre 
aquellas. Estas relaciones transfronterizas marcadas por una cultura, identidad étnica y prácticas comunes en torno a la tradición andina y a los ciclos mineros (salitre, plata, estaño), tienen antecedentes desde la época colonial (Ovando y González 2014; González 2006), cuestión destacada por varios especialistas que han detectado una resistencia local a los poderes centrales, en pos de una articulación regional transandina (Galdames y Ruz 2010; Castro 2005, 2005b), encontrando ejemplos desperdigados durante todo el siglo XX.

La lógica de las relaciones transfronterizas presentes en esta triple frontera sostenemos puede sustraerse de estos embates diplomáticos y plantear un mayor compromiso con el otro (para)diplomático -al punto que definen su identidad recíprocamente como sujetos habitantes de ciudades trasfronterizas- por no estar constreñidos por las rutinas diplomáticas, toda vez que los encuentros informales "son más abiertos para la autorrevelación... permitiendo a los participantes el poder liberarse ellos mismos de sus propias creencias estereotipadas", consecuentemente permitiendo que asuntos políticos sustanciales puedan ser abordados de una manera más constructiva y de una manera recíprocamente satisfactoria" (Cornago 2013:8). Esta premisa se nutre, además, de varias particularidades que resultan importantes tener en cuenta a la hora de analizar los actores desde la perspectiva del pluralismo diplomático:

Un intercambio comercial histórico entre Tacna y Arica que se proyecta hasta hoy (Graña, 2001); el desarrollo de localidades como Sama y Camarones antes del establecimiento de la frontera definitiva entre Chile y Perú en 1929, en donde comerciantes, arrieros, mineros, agricultores, pastores e indígenas, vieron tensionadas sus prácticas sociales y culturales, producto de la acción del Estado que buscaba establecer fronteras definitivas (González y Ovando 2017); la concreción de estrategias de desarrollo transfronterizas con resultados dispares en la subregión (Castro 2005, 2005b); la participación de estas localidades en la cooperación internacional al desarrollo, percibiendo importantes recursos por medio de estas gestiones ${ }^{15}$ (Marteles, 2009); la vigencia de intercambios culturales de variada índole entre las mismas ${ }^{16}$; la emergencia de procesos migratorios transfronterizos, que conllevan la formación de nuevas identidades asociadas a la movilidad circulatoria (Tapia 2014, 2015), entre otros.

Con todo, nuestros países y principalmente regiones epicentro del conflicto han vivido siempre aquejadas por "una relación fluctuante, afectada permanentemente por la herencia del pasado. $\mathrm{La}$ Guerra del Pacífico y sus consecuencias más visibles" (Milet 2004: 228-235). Por ello, la paradiplomacia -desde el enfoque teórico que proponemos- está llamada a ocupar en este ámbito un rol relevante que contribuya a supera y morigerar los efectos nocivos de controversias diplomáticas que aquejan a Chile y Perú ${ }^{17}$. No obstante, para ello debe contemplarse la discusión acerca de la pertinencia de incluir una lógica de arriba hacia abajo en la puesta en marcha de políticas fronterizas.

A continuación nos referiremos a propuestas singulares de las relaciones peruano-chilenas en el ámbito de la paradiplomacia según el enfoque reseñado y al que adherimos. Destacamos iniciativas locales (ariqueñas y tacneñas) ante las repercusiones del fallo de La Haya y algunas expresiones de municipalismo transfronterizo.

Un ejemplo del primer punto fue una inédita reunión entre juntas de vecinos de Tacna y Arica, -que reunió a 80 dirigentes vecinales-en el marco del primer Encuentro de Dirigentes Vecinales de Arica-Tacna Construyendo vecindad en las Fronteras. Instancia auspiciada por la Comunidad Andina de Fomento, CAF, y apoyado por la Subcomisión de Desarrollo Social y Género del Comité de Integración y Desarrollo Fronterizo de Perú y Chile. Estas, reunidas en Pachía, Tacna, redactaron el "Manifiesto Dirigentes Vecinales Tacna y Arica, Pachía, 18 de enero de 2014", en cuyas páginas se señala "Exigimos una política de Estado para la Integración Fronteriza, con una mirada local que fortalezca la convivencia" (2014:1). También señalaron "Llamamos a seguir abriendo de espacios de integración, más allá de dificultades, para así continuar el camino hacia el buen desarrollo de nuestras ciudades.

En el tercer encuentro (2do se realizó en mayo) del mimo proyecto, realizado en octubre del mismo año, se señala: "se debe acercar más aún el trabajo entre juntas vecinales de ambas ciudades, por ello 
se llevará a cabo el acto de hermanamiento de juntas de vecinos de Arica y Tacna, con la participación de los alcaldes y sus representantes de las Municipalidades de Arica, Tacna y Calana, para continuar trabajando y apoyándose mutuamente y cotidianamente. De esta forma se pretende establecer una línea de cooperación constante entre vecinos y vecinas de la zona fronteriza del sur de Perú y norte de Chile, de forma de promover las buenas relaciones humanas y la cultura de la paz más allá de cualquier conflicto diplomático gubernamental". (El Puerto Libre, 24 de octubre de 2014). Desde esta misma perspectiva, también destacamos varias instancias realizadas por el Consejo Binacional de la Juventud de Tacna y Arica, agrupación creada también dentro de la Subcomisión de Desarrollo y Género que forma parte del Comité de integración Chile-Perú (Roque y Malla, 2015). Esta instancia binacional, con el apoyo de los consulados de Chile en Tacna y Perú en Arica, han promovido una acercamiento inédito entre las sociedades civiles ${ }^{18}$ de ambas ciudades, sobre todo ad portas el fallo de La Haya. Conjeturamos que implica una instancia novedosa de resignificación de la actividad consular, siguiendo la perspectiva antropológica de Neumann (2002) el trabajo cotidiano de los diplomáticos, que busca examinar "aspectos de las prácticas diplomáticas que no dejan huella en la documentación manejada habitualmente por los historiadores" (Sanz 2015:700). O de otra forma, las prácticas que emprenden el Consejo de la Juventud y los respectivos consulados podemos interpretarlas desde una investigación etnográfica de las rutinas diplomáticas, desde la premisa de Neumann que señala que "cualquier observador que trate la debida coherencia entre las prácticas diplomáticas y los discursos debería considerar seriamente la multiplicación de intervenciones subestatales y de la sociedad en el ámbito diplomático (Neumann 2002: 627, citado por Cornago, 2015:26).

Este consejo tiene como fin "....ser un ente que trabaje por la integración y la paz entre las dos naciones y con esto propiciar la participación de la juventud en el desarrollo político, social, económico y cultural de nuestros países" (Roque y Malla 2015). De sus actividades se destacan ciertas iniciativas sociales y educativas realizadas allende la frontera y con la colaboración del consulado respectivo, así como el Encuentro Binacional de Integración Medio Ambiental realizada en Tacna,
Operativo Social Bifronterizo y el Festival Cultural Juvenil Bifronterizo. Todas ellas son concebidas como acciones de reconciliación. Al respecto implícitamente consideran dinámicas que aluden a las consecuencias de la Guerra del Pacífico, que afectaron a las poblaciones de estas ciudades por décadas, una vez finalizado el conflicto: "Nosotros no tenemos la 'culpa', pero asumimos activamente la responsabilidad que nuestros pueblos y países cometieron antes de que naciéramos. Asumimos la responsabilidad que compete a nuestros antepasados" (Roque y Malla 2015).

Sin embargo, después de La Haya las relaciones diplomáticas entre Perú y Chile han caído en un punto muerto, cuando se esperaba un acercamiento debido a que -como lo habían anunciado diferentes agentes peruanos- el límite marítimo era el último residual pendiente en la frontera entre ambos países, empero, rápidamente emergió un residual del residual: el triángulo terrestre. Al parecer agotadas las demandas tangibles, Perú comenzará a crear demandas intangibles a Chile. La opinión del jurista peruano sobre el "triángulo terrestre", Fabián Novack, es lapidaria:

El texto mismo del fallo establece claramente que el límite terrestre ha sido establecido por el tratado de Lima de 1929 y, por tanto, no se pronuncia sobre este tema. Seguirá siendo de soberanía peruana. La interpretación del presidente Piñera es unilateral y se basa en algo falso que Chile ha hecho desde el 2001, cuando construyó una caseta aduciendo que ese espacio era chileno. Para el Perú no hay más disputas pendientes, la frontera terrestre fue cerrada en 1929 y la marítima el pasado lunes 27 (Rojas, 2014).

Finalmente, otro ejemplo distinto -a nivel de universidades- lo encontramos con la puesta en marcha de un diplomado orientado al estudio de la integración fronteriza y las políticas sociales entre Tacna y Arica (UTA y la Universidad Privada de Tacna), cuyo programa será elaborado además por la Corporación de Formación Laboral al Adolescente, (CORFAL), ONG que interactúa con adolescentes y sus familias en situación de riesgo y vulnerabilidad social, de la región Arica y Parinacota. El curso busca “ abordar las temáticas pertinentes para un público amplio relacionado al trabajo social comunitario, es decir, profesionales y técnicos que se desarrollen en ciudades comprendidas en la macrorregión centro sur andina" (Universidad de Tarapacá, 5 de mayo de 2015). 


\section{Conclusiones}

Algunos incidentes diplomáticos chilenoperuanos, que tuvieron respuesta en las sociedades de Tacna y Arica de las posguerra del Pacífico, los consideramos como argumentos para intentar afirmar la hipótesis que señala que, pese a este contexto beligerante, prevaleció un periodo de la normalidad en la dinámica transfronteriza de este territorio, que comparten el norte de Chile y el Sur del Perú. Esta normalidad que se manifiesta mediante expresiones paradiplomáticas y que intentamos extrapolar al periodo de posfallo de La Haya que afectó a este espacio.

De la mano de este argumento, también planteamos que las diplomacias de los dos países -por la naturaleza de su quehacer-, si bien pueden pensar de soluciones a litigios y conflictos en forma bilateral, sus protocolos y constricciones propias de las rutinas diplomáticas han llevado a amplificar las consecuencias negativas del fallo de la CIJ de manera artificial, sobre todo ante la opinión pública regional. También se aprecia que a partir del fallo han prevalecido varias medidas complementarias de forma unilateral. Sin embargo, la paradiplomacia, también por su naturaleza informal, la que en el caso de Chile y Perú no responde a ningún marco jurídico exhaustivo y más bien se ha ido acomodando al trabajo de las sociedades civiles y los respectivos consulados, ha demostrado a partir de los casos descritos que sus encuentros informales han posibilitado a sus participantes el poder liberarse de sus propias creencias estereotipadas, consecuentemente permitiendo que asuntos políticos sustanciales puedan ser abordados de manera recíprocamente satisfactoria.

En efecto, con estos ejemplos intentamos revelar una visión distinta a cómo se interpreta la tradicional actividad política-diplomática, que han sostenido históricamente autoridades chilenas y peruanas, la que, desde sus actuaciones, no considera sus repercusiones sociales ante el surgimiento de litigios, muchos recogidos por las respectivas historias nacionales. Pues, desde esta perspectiva pluralista no figuran directamente gobiernos, ministerios de relaciones exteriores; en cambio, rescatamos experiencias sociales, "reales", que, pese a no ser realizadas por dichos actores -aunque algunas en colaboración con autoridades-, tienen relevancia política inmediata en nuestra vida cotidiana en tanto sociedades que comparten una frontera. Particularmente, esta mirada nos permite un espacio para el entendimiento político entre nuestras sociedades.

\section{Agradecimientos}

Resultado de proyecto FONDECYT No 1160209.

\section{Referencias Citadas}

Aldecoa F, y Keating, M (eds.)

1999 Paradiplomacyin Action. The Foreign Relations of Subnational Governments, Londres, Frank Cass.

Amilhat Szary, A, L.

2016 "Gentes y agentes, condiciones paradiplomáticas de la creación de una frontera móvil", en: Sergio González, Noé Cornago y Cristian Ovando (Edit.), Relaciones transfronterizas y paradiplomacia en América Latina: Aspectos teóricos y estudios de casos (pp. 47-72). Santiago de Chile: Editorial RIL.

Basadre, J.

1975 La vida y la historia. Ensayo sobre personas, lugares y problemas. Fondo del Libro BIP, Lima.

Boisier, $\mathrm{S}$.

2003 "Globalización, geografía política y fronteras". Anales de Geografía de la Universidad Complutense 23: 21-39.

Carreon, $\mathrm{P}$.

2007 Paradiplomacia y su desarrollo en el mundo. El fenómeno de los gobiernos no centrales activos internacionalmente, Revista Protocolo, Foreign Affairs \& lifestyle online, en http:// www.protocolo.com.mx/autores.php?id_ autor=65\&all_articles $=1$.
Castro, L.

2005 Regionalismo y desarrollo regional: debate público, proyectos económicos y actores locales (Tarapacá 18801930). CEIP Ediciones, Viña del Mar.

Castro, L.

2005b "Los ariqueños, el desarrollo regional y las propuestas del Comité Pro-resurgimiento de Arica, 1932”. Diálogo Andino 25: 77-99.

Cobino, $\mathrm{M}$.

2005 "La diplomacia regional. El caso italiano". Trabajos y Ensayos 2, España, Universidad del País Vasco.

Comisión Asesora Presidencial en Descentralización y Desarrollo Regional, 2014.

Constantinou, C. y Der Derian, J.

2010 Sustainable Diplomacies. Londres, Palgrave.

Constantinou, C.

2013 "Between Statecraft and Humanism: Diplomacy and Its Forms of Knowledge. International Studies Review 15: 141-162.

Cornago, $\mathrm{N}$.

2009 "La diplomacia como heterología" Universidad del País Vasco. Manuscrito en posesión del autor. 
Cornago, $\mathrm{N}$.

2013 Plural Diplomacies: Normative Predicaments and Functional Imperatives. Martinus Nijhoff Publishers, Amsterdam.

Cornago, $\mathrm{N}$.

2013b Diplomacy decentralized subnational politics and the making of sustainable diplomatic couples in Latin America. In Building Sustainable International Couples: Critical Components of a Strategy Towards Peaceful and Constructive Cooperation. Edited by B. Vassort-Rousse. Basingstoke, Palgrave.

Cornago, Noé

2014 "Beyond the diplomatic incident". In: International Colloquium Form(s) of diplomacy 12-13 June 2014, University of Toulouse 2 Le Mirail, France.

Cornago, $\mathrm{N}$.

2016 Diplomacia como heterología: pluralismo social y múltiples mediaciones institucionales en la frontera. En: Relaciones transfronterizas y paradiplomacia en América Latina: Aspectos teóricos y estudio de casos, editado por: S. González, N. Cornago y C. Ovando, 17-46. RIL editors, Santiago de Chile.

Cornago, $\mathrm{N}$.

2016b "Diplomatic Knowledge". In: The SAGE Handbook of Diplomacy, edited by Co Constantinou, P Kerr and P Sharp, 133-141. Sage Publications: London.

Der Derian, J.

1987 On Diplomacy: A Genealogy of Western Estrangement. Blackwell, Oxford.

Dilla, $\mathrm{H}$.

2016 Chile y sus fronteras: notas para una agenda de investigación. Polis (Santiago), 15(44):309-327.

Duchacek, I.

1986 The Territorial Dimension of Politics, Within, Among and Across Nation, Boulder, Westview Press.

Duchacek, I

1984 The International Dimension of Subnational SelfGovernment, The Journal of Federalism, 14(4): 5-31.

Durán, M.

2012 The Transformation of Foreign Policy and Diplomacy Bridging the gap between self and other? Mediterranean paradiplomacy as homo-diplomacy. European Consortium of Political Research, ECPR Antwerp Joint Research Sessions, University of Antwerp, Belgium 10th-15th April 2012.

Galdames, L y Ruz, R.

2010 "La Junta de Adelanto de Arica y John V. Murra. Dos lecturas sobre el desarrollo andino en el norte de Chile". Chungara, Revista de antropología chilena 42 (1): 257-270.

Gallardo, A.

2006 Paradiplomacia. La dimensión subnacional de las relaciones internacionales. Tesis para optar el grado de Magíster en Estudios Internacionales, Universidad de Chile, Santiago de Chile.

García, V.

2015 Territorios fronterizos. Agenda de seguridad y narcotráfico en Chile: el Plan Frontera Norte Estudios Internacionales 16 (31): 117-148.

González, S.

2004 "Pax castrense en la frontera norte. Una reflexión en torno a la post-guerra del Salitre: el conflicto por TacnaArica y Tarapacá". Universum19: 28-57.
González S.

2008 La llave y el candado: el conflicto entre Perú y Chile por Tacna y Arica (1883-1929). LOM Ediciones, Santiago de Chile.

González, S.

2006 Arica y la triple frontera. Integración y conflicto entre Bolivia, Perú y Chile. Aríbalo, Iquique.

González S. y Ovando, C.

2017 "Sama y Camarones, las fronteras que no fueron entre Perú y Chile". Revista de Geografía Norte Grande 66:61-82.

Graña, F.

2001 Cambio Casera: Comercio y Relaciones Fronterizas en el Extremo Norte de Chile. IV Congreso Chileno de Antropología, Colegio de Antropólogos de Chile A.G., Santiago de Chile.

Luna, J. C. y Ballesteros, H.

2005 Actualidad de los hermanamientos mexicanos. Revista Mexicana de Política Exterior 74:11-38.

Marteles, Silvia

2009 Cooperación transfronteriza en la Triple Frontera de Bolivia-Chile-Perú. En Cooperación transfronteriza e Integración en América Latina, editado por J.L. Rhi-Sausi y D. Conato, 175-215: IILA/CeSPI, Roma.

Martínez, J.

1997 “Aula Viva en la Frontera Colombo Venezolana”. Aldea Mundo 2 (3).

Milet, $\mathrm{P}$.

2004 "Chile-Perú: Las dos caras de un espejo". Revista de Ciencia Política 24(2):228-235.

Ministerio de Relaciones Exteriores de Perú

2016 "Perú y Chile realizan reunión sobre integración fronteriza", Nota de Prensa, num. 037-10/05/2016.

Ovando, C y González, S.

2014 La relación bilateral chileno-boliviana a partir de las demandas tarapaqueñas: aproximación teórica desde la paradiplomacia como heterología. Estudios Internacionales 46(177): 35-64.

Ovando, C.

2013 Algunos aspectos del desarrollo de la paradiplomacia en América del Sur vistos desde el caso chileno. Trabajos y Ensayos 16, España, Universidad del País Vasco.

Palacios R.

1976 El Perú Republicano y moderno, 1868-1968. Ediciones Studium, Lima.

Panty Neyra, O.

1999 Historia de la prensa escrita en Tacna. Edit. Javier Flores Arocutipa, Tacna.

Panty Neyra, Oscar et al.

2001 Nueva Historia General de Tacna. EPF Impresores, Tacna

Rhi- Sausi, J y Oddone, N.

2013 "Integración regional y cooperación transfronteriza en los nuevos escenarios de América Latina", Investigación \& Desarrollo 21(1):58-285.

Roque, J y Malla L.

2015 Consejo Binacional de la juventud Tacna-Arica: lineamientos y objetivos de la organización. Ponencia presentada en XI seminario bilateral en el marco del proyecto "Generación de diálogo entre Chile y Perú", Instituto de Estudios Internacionales de la Universidad de Chile (IEI), Instituto de Estudios Internacionales de la Pontificia Universidad Católica del Perú (IDEI) y (KAS) Fundación Konrad Adenauer, Arica, Chile, 5 y 6 de junio. 
Rosenblitt J.

2010 "El comercio tacnoariqueño durante la primera década de vida republicana en Perú, 1824-1836." Revista Historia, 43(1): 79-112.

Sanz, C.

2015 Agentes, redes y culturas: senderos de renovación de la historia diplomática. Actas del XII Congreso de la Asociación de Historia Contemporánea: pensar con la historia desde el siglo XXI, 687-706. Ediciones de la Universidad Autónoma de Madrid, Madrid.

Schmitt C.

2005 El concepto de lo político. Alianza Editorial, Madrid. Skuban, W.

2007 Lines in the Sand. Nationalism and Identity on the Peruvian-Chilean Frontier. Albuquerque: University of New Mexico Press.

SUBDERE

2008 Actualidad de las relaciones paradiplomáticas y de Ccooperación descentralizada de los municipios chilenos, Unidad de Relaciones Internacionales de la subdere, Ministerio del Interior de Chile, Santiago.

Tapia, M.

2014 Extranjeros fronterizos en las regiones extremas de Chile: entre migración y circulación. 1990-2014. En Extranjeros fronterizos en las regiones extremas de Chile: entre migración y circulación. 1990-2014, editado por N. Rojas y J. Vicuña, 31-55. Santiago: Ciudadano Global /OIM.

Tapia, M.

2015 Frontera, movilidad y circulación reciente de peruanos y bolivianos en el norte de Chile. Estudios atacameños. Arqueología y Antropología Surandinas, 50:195-213.

Ugalde A.

2005 "La acción Exterior de los Gobiernos no Centrales en la Unión Europea Ampliada”, en: Cursos de Derecho Internacional y Relaciones Internacionales de VitoriaGasteiz, Universidad del País Vasco, Vitoria/España.

\section{Fuentes}

\section{Prensa en línea y escrita}

Perú lanza decreto que declara al 2016 como el año de la consolidación del Mar de Grau (viernes 1 de enero de 2016). Emol.com. Recuperado de: http://www.emol.com/noticias/ Internacional/2016/01/01/766400/Peru-declara-2016-comoel-ano-de-la-consolidacion-del-Mar-de-Grau.html

"Presidente de Perú defiende creación de distrito tras crítica de ministro Gómez", sección internacional (lunes 28 de diciembre de 2015), Emol.com. Recuperado de: http:// www.emol.com/noticias/Internacional/2015/12/28/765798/ Presidente-de-Peru defiende-creacion-de-distrito-tras-criticade-ministro-Gomez.html

"Alto contingente policial resguarda la frontera entre Perú y Chile para evitar marcha al triángulo terrestre", sección nacional, (jueves 27 de agosto de 2014). La Tercera. Recuperado de: http://www.latercera.com/noticia/ nacional/2014/08/680-593185-9-en-vivo-alto contingentepolicial-resguarda-la-frontera-entre-peru-y-chile-para.shtml

"Juntas de vecinos de Arica y Tacna se hermanarán en encuentro por la integración" (24 de octubre de 2014). El Puerto Libre, espacio ciudadano. Recuperado de: http://www. elpuertolibre.cl/archivos/40663

Rojas, R. (30 de enero de 2014). "Post fallo de La Haya: Un mar de desafíos", Correo, sección política. Recuperado de: http://diariocorreo.pe/politica/ post-fallo-de-la-haya-un-mar-de-desafios-53229/

\section{Sitios web}

Comunidad Andina de Naciones, [Publicación Digital] Centros Binacionales de Atención en Frontera (CEBAF), Sitio web: http://www.comunidadandina.org/Seccion.aspx $? \mathrm{id}=123 \& \mathrm{ti}$ po $=$ TE $\&$ title $=$ centros-binacionales-de-atencion-en-fronteracebaf, consultada el 14 de abril de 2015

Comunidad Andina de Naciones, [Publicación Digital] Banco de Proyectos de Integración y Desarrollo Fronterizo (BPIF). Sitio web: http://www.comunidadandina.org/Seccion.

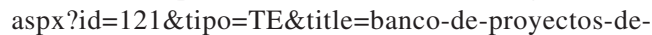
integracion-y-desarrollo-fronterizo-bpif, consultada el 14 de abril de 2015

Comunidad Andina de Naciones, [Publicación Digital] Zonas de Integración Fronteriza (ZIF). Sitio web: http://www.comunidadandina.org/Seccion. aspx $? i d=122 \&$ tipo $=$ TE \& title=zonas-de-integracionfronteriza-zif, consultada el 14 de abril de 2015

Corporación de Formación Laboral al Adolescente (CORFAL). Sitio web: http://www.corfal.cl/quienes-somos/, consultada el 14 de abril de 2015.

Universidad de Tarapacá,2015, [Publicación Digital] “Universidad de Tarapacá firma importante convenio de ejecución de Diplomado para el estudio de la Integración Fronteriza y las Políticas Sociales", 5 de mayo de 2015.Fuente: http://www. uta.cl/universidad-de-tarapaca-firma-importante-convenio-deejecucion-de-diplomado-para-el-estudio-de-la-integracionfronteriza-y-las-politicas-sociales/web/2015-05-05/161736. html, consultada el 14 de abril de 2015.

\section{Notas}

1 La prensa chilena destaca ambos hitos: "Perú lanza decreto que declara al 2016 como el 'año de la consolidación del Mar de Grau"'. La medida fue adoptada con el objetivo de destacar la importancia del triunfo obtenido en el juicio contra Chile en la Corte Internacional de Justicia de La Haya en 2014. Un decreto supremo de la Presidencia del Consejo de Ministros (PCM) publicado este viernes en el diario oficial El Peruano señaló que todos los documentos oficiales del Gobierno estarán encabezados por esa frase. El
Mar de Grau es la denominación aprobada por el Congreso peruano para nombrar las aguas jurisdiccionales de Perú en el océano Pacífico, en referencia al almirante Miguel Grau, héroe de la Guerra del Pacífico (1879-1883) (Emol, viernes 1 de enero de 2016).

En cuanto a las controversias por la creación de la nueva unidad político administrativa: "El Presidente del Perú, Ollanta Humala, afirmó hoy que la creación del distrito peruano en la zona fronteriza con Chile es 'irreversible', 
tras las declaraciones del ministro chileno de Defensa, José Antonio Gómez, quien consideró el acto de una 'entelequia jurídica' (...). El distrito peruano se ubica en la zona denominada 'triángulo terrestre', un territorio de unos 3,7 kilómetros cuadrados cuya soberanía reclaman ambos países" (Emol.com, lunes 28 de diciembre de 2015).

2 Los historiadores peruanos tacneños reconocen periodos diferenciados en esos cuarenta y cinco años de la administración chilena de ese territorio. Raúl Palacios nos habla de una "chilenización pacífica" hasta 1900, luego vendría una etapa de "chilenización violenta" (1974:63). Por su parte, Oscar Panty Neira identifica una "chilenización conciliadora", entre 1884 y 1900, le seguiría una fase de “chilenización violenta", 1900-1922, y una última fase de “arbitraje y negociaciones", 1922-1929. Este autor prosigue, acertadamente, la periodización, señalando uno de "crisis económico-social", 1929-1939, otro de "estabilidad y postergación”, 1940-1945, etc. (1999). Es evidente que estas etapas están relacionadas con la política exterior bilateral peruano-chilena. Sin embargo, la narrativa de los historiadores peruanos se enfoca solamente en una política estatal chilena respecto de esos territorios, dejando -por omisión- el supuesto que no hubo una política peruana o esta habría sido solo reactiva.

3 Posiblemente haciendo una no explícita referencia a la obra de teatro de Mario Vargas Llosa.

4 Sostenemos que durante todo el periodo 1884-1929, existieron redes sociales y vida cotidiana que sostuvieron o amortiguaron el conflicto, evitando el casus belli, donde algunos actores tuvieron un papel paradiplomático clave y ciertas estructuras sociales permitieron ese papel, entre ellos, las cofradías religiosas, los comerciantes transfronterizos, maestros primarios, etc.; y entre las estructuras tenemos a los linajes andinos transfronterizos que permitieron la existencia de redes familiares en Tacna y Arica, el mercado tradicional y las costumbres andinas, etc.

5 El 27 de agosto de 2014, Ciro Silva Paredes, coordinador del Comité Cívico Patriótico de Tacna convocó a una marcha desde la ciudad de Tacna al dyenominado "triángulo terrestre", con la intención de simbólicamente reivindicar su soberanía. Si bien no convocó a más de 100 persona, la marcha tuvo una destacable cobertura mediática por ambos países, puesto que ésta, que finalmente fue detenida, debía cruzar territorio actualmente de soberanía chilena. El diario La Tercera de Chile cubrió así el episodio:

“... alto contingente policial resguarda la frontera entre Perú y Chile para evitar marcha al triángulo terrestre. Tanto en Arica como en Tacna se ha implementado un plan especial de seguridad para evitar desórdenes tras el llamado de un grupo nacionalista peruano a marchar hacia el denominado 'triángulo terrestre"” (La Tercera, 27 de agosto de 2014).

6 Se trata de 51 municipios del sur del Perú, norte de Chile y del centro oeste de Bolivia que se asociaron desde el año 2001 para concretar proyecto de desarrollo trinacional, con el apoyo de organismos internacionales tales como el Banco Interamericano de desarrollo y el Cespi. Véase: http://www.aymarasinfronteras.org/

7 Este debate lo comenzaron la teorías de las relaciones internacionales posmodernas, centrando su análisis "en la superación del Estado nación y la emergencia de nuevas formas de gobernanza, resultantes de la interacción no-jerárquica y flexible de una multiplicidad de redes no estatales" (Ugalde, 2005: 297).Dentro de este marco interpretativo, surge la genealogía de la diplomacia. Esta corriente profundiza la idea de la paradiplomacia entendida como vínculos internacionales entre actores sociales pre estatales, los que siempre han existido, pero han sido invisibilizados por el Estado (Aguirre, 2001; Cornago, 2016).

8 Este enfoque teórico se contrapone a las visiones racionalistas de la diplomacia que explican el intercambio diplomático como un mero juego de actores que busca la conciliación de intereses-valores o desde la manipulación o instrumentalización del otro (una relación sujeto-objeto) para alcanzar mi objetivo en clave Razón de Estado. Estas posibilidades de los estudios diplomáticos, desde un criterio epistemológico, Cornago (2016b:136), siguiendo a Constantinou, lo resume así: "cualquier concepción del conocimiento diplomático debería tomar en cuenta las experiencias de los diplomáticos con el compromiso del "otro diplomático" , ya sea considerado como 'objeto' puesto en una posición observable desde fuera de las encerradas fronteras del sí mismo o alternativamente como un 'sujeto' cuya sola presencia involucra el verdadero cuestionamiento del propio ser en un proceso de reconfiguración de subjetividades mutuas".

9 En efecto, las Unidades Regionales de Asuntos Internacionales (URAI) en 2005 empezaron a funcionar y, por diversas razones, han enfrentado dificultades para consolidar una estructura y trabajo estable. Inicialmente se sugiere que la orientación estratégica de estas unidades se oriente en cuatro líneas de acción principales: a) Acciones de Integración priorizando gobiernos subnacionales de países vecinos: Argentina, Bolivia, Brasil Paraguay, Perú. b) Acciones de Cooperación Internacional enfatizando dimensiones económicas, social, cultural, turística y, entre otras, científico-tecnológicas. c) Acciones de promoción de inversiones extranjera en la región y posicionando a productores locales en nuevos mercados en América Latina y otros contextos. d) Acciones de Participación Ciudadana que permitan incorporando a la ciudadanía en acciones que favorezcan la diplomacia social (COMISIÓN ASESORA, 2014:55-56).

10 Desde el año 2003 existe una unidad dentro de la cancillería, la DICORE (2016), que coordina la acción exterior de las regiones. La Dirección de Coordinación Regional es responsable de apoyar y coordinar a las regiones y municipios del Chile en la realización de sus proyectos e iniciativas en el ámbito internacional. Para ello, la dicore permite vincular a los gobiernos regionales y locales con las direcciones del Ministerio de Relaciones Exteriores y la red de embajadas, misiones y consulados Ver: http:// www.minrel.gov.cl/prontus_minrel/site/artic/20080903/ pags/200809031113 14.php\#T0)

11 Sus iniciativas han tendido a visibilizar la acción exterior de las regiones y comunas a través de colaboración técnica, sistematización y cuantificación de las actividades y gestiones emprendidas por distintos actores regionales y comunales. Adicionalmente, el organismo público ha planteado la necesidad de generar una mayor supervisión y coordinación por parte del gobierno central hacia las actividades internacionales emprendidas por las comunas, 
a objeto de mantener coherencia con la política exterior del país; para lo cual ha instado a la consideración de alguna modificación legal que permita el ordenamiento de la acción paradiplomática y de cooperación descentralizada en Chile a partir de la experiencia y práctica internacional (Subdere, 2008).

12 Basta con consultar el sitio web de DICORE, donde se aprecia que su información al presente año (2017) "Documentos de apoyo" es del año 2004.

Consultar: http://www.minrel.gob.cl/minrel/site/edic/base/ port/coordinacion_regional.html

13 XIII Comité de Integración y Frontera Chile-Bolivia, instancia de cooperación y gestión fronteriza que reúne cada dos años a actores sociales y políticos de las regiones limítrofes de ambos países, agendado para noviembre de 2013 fue suspendido indefinidamente por el gobierno boliviano. Además la Unidad Regional de Asuntos Internacionales, URAI, del Gobierno Regional de Tarapacá pasó de tener un departamento de cooperación internacional a una oficina, luego el cargo quedó vacante y actualmente se está restituyendo.

14 Muchas veces sin considerar los intereses de las regiones afectadas por estas decisiones digitas desde las respectivas capitales, que contemplan a las regiones como apéndices y desde la perspectiva de las fronteras interiores o zonas alejadas del centro político y en donde el Estado debe cumplir un rol clave en materia de preservar la soberanía ante amenazas foráneas como el narcotráfico y otras amenazas tradicionales (García, 2015).

15 Destacamos los aportes del Cespi a través del programa Fronteras Abiertas (Marteles 2009); los aportes del Banco Interamericano de Desarrollo, entre otros.

16 En este ámbito es importante destacar el rol de las Universidades de Tarapacá y Privada de Tacna, de Arica y Tacna, respectivamente. También las iniciativas en el marco del CRISCOS, Consejo de Rectores por la Integración de la Subregión Centro Oeste de Sudamérica en que participan ambas universidades. Ver: http://www.criscos.cl/

17 Por ejemplo, ante la marcha a la frontera convocada por grupos nacionalistas tacneños ad por tas del fallo, las juntas de vecinos de Tacna y Arica se reúnen con apoyo de los consulados y comités de integración para hacer un encuentro por la paz y la vecindad transfronteriza.

18 Si bien Chile posee un marco institucional limitado para promover la acción exterior y transfronteriza de sus regiones, dentro de los desafíos para profundizar la descentralización, la comisión asesora presidencial plantea promover Acciones de Participación Ciudadana que permitan ir incorporando a la ciudadanía en acciones que favorezcan la diplomacia social. 
